\title{
Effect of mori folium and eucommiae cortex oral solution on immune function of chicken infected with IBDV
}

\author{
Haifeng YANG ${ }^{1 *}$ (D), Yi CUI ${ }^{2}$, Xiaolan $\mathrm{CHEN}^{1}$, Jie $\mathrm{HUANG}^{2}$, Chunmao JIANG ${ }^{1}$, Jingui $\mathrm{LI}^{2 *}$
}

\begin{abstract}
Sangdu oral liquid (MFEC), a kind of traditional Chinese medicine, is used as a new immune enhancer for veterinary drugs or vaccines. In current study, we aimed to evaluate the effect and mechanism of MFEC on improving the immune response of B87 vaccine. Eighty SPF chickens were divided into blank control group (BC group), infectious bursal disease virus (IBDV) group (IBDV group), vaccine group (B87+IBDV group) and vaccine and Sangdu oral liquid combination group (MFEC+B87+IBDV group). In addition to the $\mathrm{BC}$ group, all other groups were infected with IBDV to establish immunosuppressive model. Chickens in both B87+IBDV and MFEC+B87+IBDV groups were immunized with B87 vaccine. Chickens in MFEC+B87+IBDV group were given MFEC for 7 days. Compared with B87+IBDV group, there were significantly lower morbidity and mortality, higher level of antibody, less tissue damage, lower expression of Bax and higher expression of Bcl-2 of MFEC+B87+IBDV group $(P<0.05)$. In MFEC+B87+IBDV group, the expression of IBDV-VP2, IL-6 and IFN- $\gamma$ mRNA in bursal tissue was significantly lower than that in $\mathrm{B} 87+\mathrm{IBDV}$ group $(P<0.05)$. MFEC can effectively improve the immune response to $\mathrm{B} 87$ vaccine, and resist the invasion and replication of IBDV to avoid the tissue damage and apoptosis caused by IBDV infection.
\end{abstract}

Keywords: Sangdu Oral Liquid; B87 vaccine; infectious bursal disease; antibody; apoptosis.

Practical Application: The immune improvement response of B87 vaccine using MFEC.

\section{Introduction}

Infectious bursal disease virus (IBDV) infection would result in severe immunosuppression and death in chickens. Once infected, chickens showed typical clinical symptoms or even death in 3-7 days (Qin \& Zheng, 2017). The main organ for IBDV replication was the bursa of Fabricius, in which the virus invaded B lymphocytes, leading to severe atrophy of the bursal follicles and lymphocyte depletion (Ivanyi \& Morris, 1976). Avian bursa is the main site where B lymphocytes to differentiate into plasma cells under the stimulation of antigen and secrete immunoglobulin, which play a key role in the pathogenesis of autoimmune diseases (Matsushita, 2019).

Currently, vaccination is the most effective way to prevent infectious bursal disease (IBD). Based on the difference of virulence, the commonly used live vaccines are classified into medium-virulence, medium-strong virulence and weak virulence vaccines (Jackwood, 2017). The medium-virulence live vaccines could induce antibodies production rapidly and long-term protective immune responses. Although the vaccine is effective, it also leads some side effects. Previous studies have reported that the live vaccine with the medium or medium-strong virulence can lead to immunosuppression (Qin \& Zheng, 2017; Spackman et al., 2018; Dey et al., 2017). In addition, the quality of vaccines produced by various manufacturers is different, and the preservation and transportation of live vaccines is also strict. Thus, immunization error or a strong strain virus attack could lead to the disease epidemics. Once infected, there is still a huge risk on raised by production performance. Therefore, it is necessary to use immunopotentiators to reduce the risk.

Sangdu Oral Liquid (MFEC), a kind of traditional Chinese medicine, consists of mulberry leaf polysaccharide and eucommia ulmoides aqueous extract. In recent years, some researchers have proposed that MFEC can be used as a new immune enhancer for veterinary drugs or vaccines (Jia et al., 2018). However, the mechanism by which MFEC enhances the therapeutic or immune effects is still unknown. It was found that MFEC alone was not effective for treating IBD. The results showed that after treatment with MFEC, the incidence of IBD did not decrease, but the mortality was reduced, which indicated that MFEC had a certain regulatory effect on IBDV infection, and might be used as an immunopotentiator to reduce the risk of vaccine and increase the immune response of vaccine (Wang et al., 2017). Pharmacological studies showed that both mulberry leaf polysaccharides and eucommia ulmoides polysaccharides could stimulate the proliferation of lymphocytes in mouse spleen and chicken peripheral blood, and could improve the immune response to new city disease vaccine and blue ear disease vaccine in vivo (Chen et al., 2015; Delibaier et al., 2017).

Therefore, to investigate whether MFEC could improve the immune response to B87 vaccine in chicken, animal model with incomplete immunity were established and then the animals 
were infected with the IBDV-SJ strain to observe the immune potentiating effect of MFEC on the B87 vaccine.

\section{Materials and methods}

\subsection{Drugs and animals}

MFEC was provided by Jiangsu Agri-animal Husbandry Vocational College, containing $1.2 \mathrm{~g} / \mathrm{ml}$ of crude drug, 0.0343 $\mathrm{mg} / \mathrm{mL}$ of rutin and $0.119-0.131 \mathrm{mg} / \mathrm{mL}$ of rosinol diglucoside. The IBDV-JS strain was provided by Professor Qin Aijian of Yangzhou University. The medium-virulence vaccine of chicken IBDV (B87 strain) was purchased from Wuhan Zhongbo Biotechnology Co., Ltd. Eighty-one days old SPF chickens and sixty chicken embryos were purchased from Jinan Seth Poultry Company. Chicken feed was provided by the Institute of Poultry of the Chinese Academy of Agricultural Sciences and does not contain pharmaceutical additives. This study was performed under the guidelines of Jiangsu Laboratory Animal Welfare and Ethical of Jiangsu Administrative Committee of Laboratory Animals (No. SYXKSU-2007-0005)

\subsection{Virus rejuvenation and EID50}

Twenty-one days old SPF chickens was orally infected with the virus. The bursas of dead chicken were obtained and grinded with PBS, and then centrifuged for $4000 \mathrm{r} / \mathrm{min}$ at $4{ }^{\circ} \mathrm{C}$ for 10 min, then the supernatant was taken. Ten folds serially diluted rejuvenated virus solution, $10^{-1}, 10^{-2}, 10^{-3}, 10^{-4}, 10^{-5}, 10^{-6}, 10^{-7}, 10^{-}$ ${ }^{8}, 10^{-9}, 10^{-10}$, were inoculated into 9 to10-days old SPF chicken embryos at the site of chorioallantoic membrane. Each chicken embryo was inoculated with $100 \mu \mathrm{l}$ rejuvenated virus solution. Chicken embryos that died within $24 \mathrm{~h}$ were discarded. The chorioallantoic membrane of chicken embryos that died within 48 to $96 \mathrm{~h}$ was collected and ground. The virus was detected by qRT-PCR, and the $\mathrm{EID}_{50}$ was calculated as $10^{-6.784}$. The virus was inoculated with $100 \mu \mathrm{l} /$ feather according to $\mathrm{EID}_{50}$. The dose of B87 vaccine was half of the recommended dose.

\subsection{Immunization}

All chickens were kept in isolators and randomly divided into four groups, including blank control group (BC), IBDV infection control group (IBDV), B87 vaccination control group (B87 + IBDV), B87 vaccine and the Sangdu oral solution group (MFEC + B87 + IBDV) (20 chickens in each group). At the age of 19-day, chickens in B87+IBDV and MFEC+B87+IBDV groups were immunized with $\mathrm{B} 87$ vaccine, and the remaining two groups were treated with the same amount of normal saline. At the age of 22-day, chickens in MFEC+B87+IBDV group were given MFEC $(9.6 \mathrm{~g} / \mathrm{kg})$ and the other two groups were given the same amount of normal saline for 7 days. At the age of 25-day, except for the $\mathrm{BC}$ group, the other three groups were infected with IBDV by mouth and nose. The chickens were sacrificed at the age of 32-day and the serum, bursa, spleen, kidney and other organs were collected.

\subsection{Clinical observation index}

The mental state, diet, weight changes, morbidity, mortality and the typical symptoms of IBD were recorded. During the necropsy, the bursa and spleen were weighed to calculate the organ index (organ weight/body weight $\times 100 \%$ ).

\subsection{Detection of serum antibody}

At 5 days after challenge, the level of IBDV antibody in the chicken serum was detected by IDEXX kit. The absorbance at $450 \mathrm{~nm}$ (OD450) was measured using a Model 550 Microplate Reader (Bio-Rad, Hercules, CA, USA).

\subsection{Detection of Serum biochemical indicators}

Three chickens of each group were randomly selected and blood samples were taken from the wing veins. Serum biochemical indicators including alanine aminotransferase (ALT), aspartate aminotransferase (AST), total protein (TP), albumin (ALB), globulin (GLB) and other indicators were analyzed by a fully automated blood biochemical analyzer.

\subsection{Histopathological examination for the pathophysiologic conditions}

The bursa, spleen, kidney and other organs were collected and detected by conventional HE staining. The grading criteria refers to the scoring standard of Shaw et al. (Shaw \& Davison, 2000), which is, no bursal and follicular damage, clear boundary between medulla and cortex ( 0 point), mild necrosis of bursa, mild loss of lymphocytes, a small number of follicle atrophy, overall structure integrity (1 point), moderate atrophy of $1 / 3$ $1 / 2$ follicles, increased lymphocyte depletion, heterophilic cell aggregation, macrophage and epithelial reticular cell hyperplasia, a small vacuole-like structure (2 points), severely necrosis and atrophy of more than $1 / 2$ follicles, lymphocytes were depleted, and follicular structure disappeared and replaced by connective tissue and fibroids ( 3 points).

\subsection{Detection of apoptosis protein in bursa of Fabricius}

The expression site and content of Bax in the bursal tissue was detected by paraffin immunohistochemistry on serially cut tissue sections utilizing an avidin-biotinylated immunoperoxidase methodology under microscope. For each staining, appropriate positive and negative controls were performed. Immunostaining was performed with antibodies to Bax (1:30 dilution, Dako).

Western blotting was used to detect apoptosis proteins Bax and Bcl-2 in the bursa of Fabricius. The Image J image processing system was used to perform density scan on the image. The ratio of the target protein band to the gray value of the internal reference was analyzed to present the relative expression of each sample protein.

\subsection{Detection of cytokine in bursa of Fabricius}

QRT-PCR was used to detect the mRNA expression of IBDV-VP2, IFN- $\gamma$ and IL- 6 in the bursal tissue. The mRNA in bursa of Fabricella was extracted using the TRIZOL and reverse transcribed into cDNA using a reverse transcription kit. After adding the reagent according to the qRT-PCR kit (primer sequence in Table 1), the qRT-PCR system was performed according to 
Table 1. Primer sequence.

\begin{tabular}{|c|c|c|}
\hline \multicolumn{2}{|c|}{ Primer } & \multirow{2}{*}{$\frac{\text { Sequence }\left(5^{\prime}-3^{\prime}\right)}{\text { TAAGGATCCACGATCGCAGCGATGACAAACCTGCAAGAT }}$} \\
\hline IBDV-VP2 & $\mathrm{F}$ & \\
\hline \multirow{3}{*}{ IFN- $\gamma$} & $\mathrm{R}$ & TGGTCTAGATTACCTTAGGGCCCGGATT \\
\hline & $\mathrm{F}$ & AGCTGACGGTGGACCTATTATT \\
\hline & $\mathrm{R}$ & GGCTTTGCGCTGGATTC \\
\hline \multirow[t]{2}{*}{ IL-6 } & $\mathrm{F}$ & CTGCCCAAGGTGACGGAG \\
\hline & $\mathrm{R}$ & GGTAGGTCTGAAAGGCGAACA \\
\hline \multirow[t]{2}{*}{$\beta$-actin } & $\mathrm{F}$ & ATTGCTGCGCTCGTTGTT \\
\hline & $\mathrm{R}$ & CTTTTGCTCTGGGCTTCA \\
\hline
\end{tabular}

Note: $\mathrm{F}$ is the upstream primer and $\mathrm{R}$ is the downstream primer.

the following procedure: pre-denaturation at $95^{\circ} \mathrm{C}$ for $2 \mathrm{~min}$, then $95^{\circ} \mathrm{C}$ for $10 \mathrm{~s}, 60^{\circ} \mathrm{C}$ for $30 \mathrm{~s}, 40$ cycles.

\subsection{Statistical analysis}

All statistical analysis was conducted by SPSS 19.0 and Prism 6.0 statistical analysis software. Difference between groups was evaluated by one-way analysis of variance (one-way ANOVA). The results were expressed as mean \pm standard deviation. Results with $\mathrm{P}$ values of less than 0.05 were considered as significant difference.

\section{Results}

\subsection{Vaccine with MFEC reduced the morbidity and mortality of chickens}

The typical symptoms of IBD occurred in $100 \%$ chickens if the IBDV group from the second day after inoculation. On the third day, death occurred in the IBDV group, while no death was found in other three groups. On the third day, mild symptoms were recorded for individual chickens of the B87+IBDV group, with an incidence of $30 \%$. There were no symptoms found in chickens of MFEC+B87+IBDV group and the BC group.

There was no death in all groups except the IBDV group, and the mortality rate in the IBDV group was $40 \%$ (Table 2). Due to the infection of viruses and immunization of vaccines, the level of the antibody was significantly increased $(P<0.05)$ (Table 3), which might be the reason that vaccine with MFEC reduced the morbidity and mortality in chickens.

\subsection{Vaccine with MFEC inhibited the organs lesions of chicken}

The weight gain of the chickens in IBDV group were the lowest. There were no significant differences of the weight gain of chickens between the MFEC+B87+IBDV group and the $\mathrm{BC}$ group $(P>0.05)$. The weight gain of the $\mathrm{B} 87+\mathrm{IBDV}$ group was slightly lower than that of the MFEC+B87+IBDV group. The spleen index for chickens in IBDV group was significantly higher than that in the other groups $(P<0.05)$, while there was no significant difference among the other groups $(P>0.05)$.

There was no significant difference of bursa of Fabricius index between the $\mathrm{BC}$ group and the MFEC+B87+IBDV group $(P>0.05)$. Bursa of Fabricius index in IBDV group and
Table 2. Morbidity and mortality $(n=20)$.

\begin{tabular}{ccc}
\hline Group & Morbidity (\%) & Mortality (\%) \\
\hline BC & 0 & 0 \\
IBDV & 100 & 40 \\
B87+IBDV & 30 & 0 \\
MFEC+B87+IBDV & 0 & 0 \\
\hline
\end{tabular}

Note: BC, blank control group (given normal saline). IBDV, infected control group, infected with IBDV after receiving $1 / 2$ dose of $B 87$ vaccine (once). B87+IBDV, immune control group, and $1 / 2$ dose of B87 vaccine was given (once). MFEC+ B87+IBDV, combination group of vaccine and MFEC. Half dose of vaccine was inoculated (once), and $9.6 \mathrm{~g} / \mathrm{kg}$ of MFEC was given (7 days in a series), and IBDV was also infected.

the B87+IBDV group were significantly lower than that of the MFEC+B87+IBDV group $(P<0.05)$. The bursa of Fabricius of the IBDV group was atrophied, and the cavity of the bursa was filled with jelly-like, transparent and yellow liquid. Different degrees of atrophy occurred in the B87+IBDV group (Table 4).

The normal range of serum biochemical indicators for SPF chicken was obtained from previous studies (Ping, 2016; Leng et al., 2007; Zhao et al., 2018), which was ALT: 1.75-4.55 (U/L), AST: 167.8-266.4 (U/L), TP: 28.67-43.97 (U/L), ALB: 9.18-17.74 (U/L), GLB: 12.37-26.86 (U/L). The results showed that the ALT of the IBDV group was higher than normal range. Although the AST was within the normal range, it was significantly lower in the $\mathrm{BC}$ group compared with other three groups, suggesting that liver damage might occurred. There was no significant difference of levels of TP, ALB and GLB between B87+IBDV group and MFEC+B87+IBDV group, while ALB and GLB of B87+IBDV group and MFEC+B87+IBDV group were significantly higher than that of IBDV group $(P<0.05)$ (Table 5$)$.

The bursa of Fabricius in IBDV group was severely atrophied, while yellowish color, tissue elasticity loss, thin wall and yellow transparent liquid was observed. The bursa of Fabricius in the BC group and the MFEC+B87+IBDV group were milky white, while tissue with full of elasticity and no fluid in the cyst were found.

Although the bursa of Fabricius of the B87+IBDV group had no obvious lesions, the size and weight decreased significantly compared with the $\mathrm{BC}$ group $(P<0.05)$. IBDV group had the highest bursal lesion score with 3 points, while the points of the B87+IBDV group and MFEC+B87+IBDV group were lower, and the $\mathrm{BC}$ group had the lowest bursal lesion score (Figure 1, Table 6). 
Table 3. Antibody levels in serum $(\mathrm{n}=20)$.

\begin{tabular}{cccc}
\hline Group & OD value & S/P value & Antibody tier \\
\hline BC & $0.067 \pm 0.048$ & $0.016 \pm 0.006$ & $25.99 \pm 10.02^{\mathrm{d}}$ \\
IBDV & $0.401 \pm 0.025$ & $0.577 \pm 0.043$ & $1259.62 \pm 101.89^{\mathrm{c}}$ \\
B87+IBDV & $0.425 \pm 0.079$ & $0.680 \pm 0.090$ & $1506.99 \pm 216.38^{\mathrm{b}}$ \\
MFEC+B87+IBDV & $0.495 \pm 0.086$ & $0.810 \pm 0.104$ & $1821.04 \pm 253.96^{\mathrm{a}}$ \\
\hline
\end{tabular}

Note: the same shoulder marks in the same column mean no significant difference $(\mathrm{P}>0.05)$, while different shoulder marks mean significant difference $(\mathrm{P}<0.05)$.

Table 4. Change in body weight and organ index of each group $(\mathrm{n}=10)$.

\begin{tabular}{cccc}
\hline Group & Weight changes/G & Spleen index (100\%) & Bursa of Fabricius index (100\%) \\
\hline BC & $205.34 \pm 21.15^{\mathrm{a}}$ & $0.18 \pm 0.03^{\mathrm{a}}$ & $0.46 \pm 0.16^{\mathrm{a}}$ \\
IBDV & $140.26 \pm 51.29^{\mathrm{c}}$ & $0.30 \pm 0.09^{\mathrm{b}}$ & $0.26 \pm 0.06^{\mathrm{b}}$ \\
B87+IBDV & $183.04 \pm 35.06^{\mathrm{b}}$ & $0.16 \pm 0.04^{\mathrm{a}}$ & $0.30 \pm 0.16^{\mathrm{b}}$ \\
MFEC+B87+IBDV & $206.66 \pm 16.05^{\mathrm{a}}$ & $0.17 \pm 0.03^{\mathrm{a}}$ & $0.37 \pm 0.07^{\mathrm{a}}$ \\
\hline
\end{tabular}

Note: the same shoulder marks in the same column mean no significant difference $(\mathrm{P}>0.05)$, while different shoulder marks mean significant difference $(\mathrm{P}<0.05)$.

Table 5. Biochemical indicators of serum.

\begin{tabular}{ccccrr}
\hline Group & ALT(U/L) & AST(U/L) & TP(U/L) & ALB(U/L) & GLB(U/L) \\
\hline BC & $2.8 \pm 1.45^{\mathrm{b}}$ & $146.83 \pm 18.16^{\mathrm{b}}$ & $27.47 \pm 1.77^{\mathrm{ab}}$ & $9.83 \pm 0.59^{\mathrm{a}}$ & $17.63 \pm 1.18^{\mathrm{a}}$ \\
IBDV & $4.63 \pm 2.5^{\mathrm{a}}$ & $254.6 \pm 37.33^{\mathrm{a}}$ & $25.7 \pm 1.96^{\mathrm{b}}$ & $6.83 \pm 1.04^{\mathrm{b}}$ & $19.23 \pm 0.29^{\mathrm{a}}$ \\
B87+IBDV & $1.07 \pm 0.59^{\mathrm{b}}$ & $172.63 \pm 20.26^{\mathrm{b}}$ & $32.37 \pm 2.81^{\mathrm{a}}$ & $11.47 \pm 0.87^{\mathrm{a}}$ & $20.9 \pm 1.95^{\mathrm{a}}$ \\
MFEC+B87+IBDV & $1.37 \pm 0.4^{\mathrm{b}}$ & $177.03 \pm 9.21^{\mathrm{b}}$ & $31.33 \pm 2.12^{\mathrm{ab}}$ & $11.27 \pm 0.67^{\mathrm{a}}$ & $20.07 \pm 1.47^{\mathrm{a}}$ \\
\hline
\end{tabular}

Note: the same shoulder marks in the same column mean no significant difference $(\mathrm{P}>0.05)$, while different shoulder marks mean significant difference $(\mathrm{P}<0.05)$.

Table 6. Bursa of Fabricius lesion score $(n=3)$.

\begin{tabular}{lcccc}
\hline & BC & IBDV & B87+IBDV & MFEC+B87+IBDV \\
\hline Bursa of Fabricius lesion score $(1 \sim 3)$ & 0 & $3^{\text {a }}$ & $1.67 \pm 0.57^{\mathrm{b}}$ & $0.33 \pm 0.58^{\mathrm{c}}$ \\
\hline
\end{tabular}

Note: the same shoulder marks in the same column mean no significant difference $(\mathrm{P}>0.05)$, while different shoulder marks mean significant difference $(\mathrm{P}<0.05)$.

Pathological sections of the spleen showed that the white pulp, central artery, and arterial lymph sheath structure were clear and intact in the $\mathrm{BC}$ group. The white pulp structure of the IBDV group was destroyed and a large number of vacuoles were formed. It could be observed that the spleen tissues of B87+IBDV group and the MFEC+B87+IBDV group were dense, no vacuole-like structure was observed. Diffuse red bleeding was found in the B87+IBDV group, but no bleeding was found in the MFEC+B87+IBDV group (Figure 2).

In the IBDV group, the renal tubules space was enlarged and filled with blood cells, and the renal capsule was slightly condensed. In the B87+IBDV group and the MFEC+B87+IBDV group, the renal tubules were closely arranged, the structure was intact, and the renal capsules were normal (Figure 3).

\subsection{Vaccine with MEFC inhibit the apoptosis in bursa of Fabricius after infection}

The expression of Bax in bursa of Fabricius of $\mathrm{BC}$ group was relatively low. In the IBDV group, a large number of apoptotic cells in the follicles were observed, and the bursal tissue was severely damaged. A large number of voids appeared in the follicles of the chicken in B87+IBDV group, while the Bax expression was observed in the intercellular space. No obvious apoptosis was observed in the follicles of MFEC+B87+IBDV group (Figure 4).

Western Blot detection showed that the expression of Bax in bursa of Fabricius in MFEC+B87+IBDV group decreased significantly compared with $\mathrm{B} 87+\mathrm{IBDV}$ group $(P<0.05)$. The expression of $\mathrm{Bcl}-2 / \mathrm{Bax}$ in IBDV group was significantly lower than that in BC group and B87+IBDV group. Vaccine with MEFC might inhibit apoptosis (Figure 5).

\subsection{Vaccine with MFEC reduced the expression of IBDV- VP2, IFN- $y$ and IL-6 mRNA in bursa of Fabricius after infection}

The expression of IBDV-VP2 of in IBDV group was significantly higher than that in the other groups $(P<0.05)$. There was no difference of the expression of IBDV-VP2 between the MFEC+B87+IBDV group and the $\mathrm{BC}$ group, while the expression of IBDV-VP2 was significantly lower than that in B87+IBDV group $(P<0.05)$. The same trend was also found in the mRNA expression of IFN- $\gamma$ and IL-6 (Figure 6). 

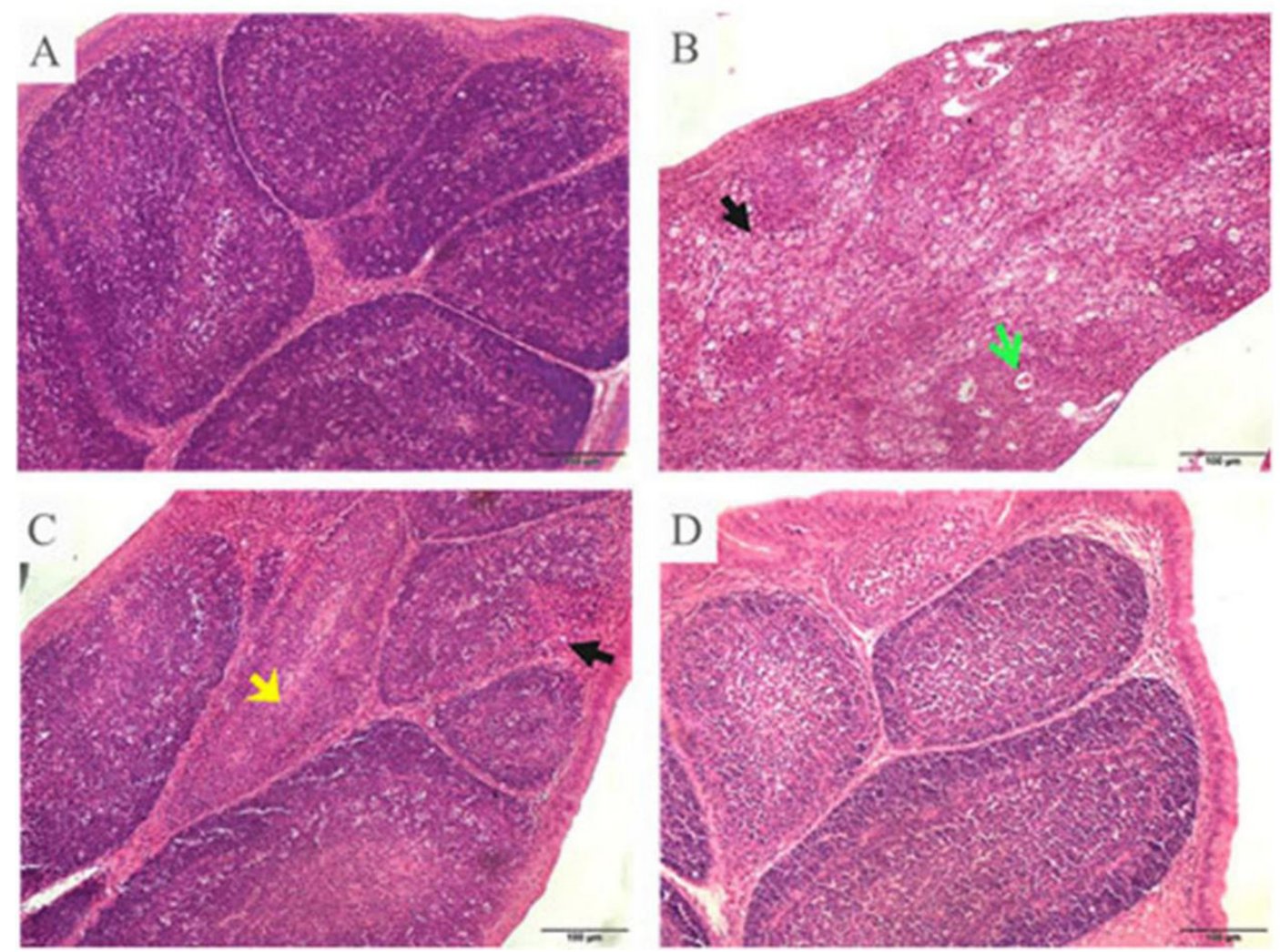

Figure 1. Pathological section of bursa of Fabricius (200x). (A) BC group; (B) IBDV group; (C) B87+IBDV group; (D) MFEC+B87+IBDV group. Green arrow is vacuolar necrosis. Black arrow is atrophied and damaged follicles. Yellow arrow is interstitium widened, fibrotic.
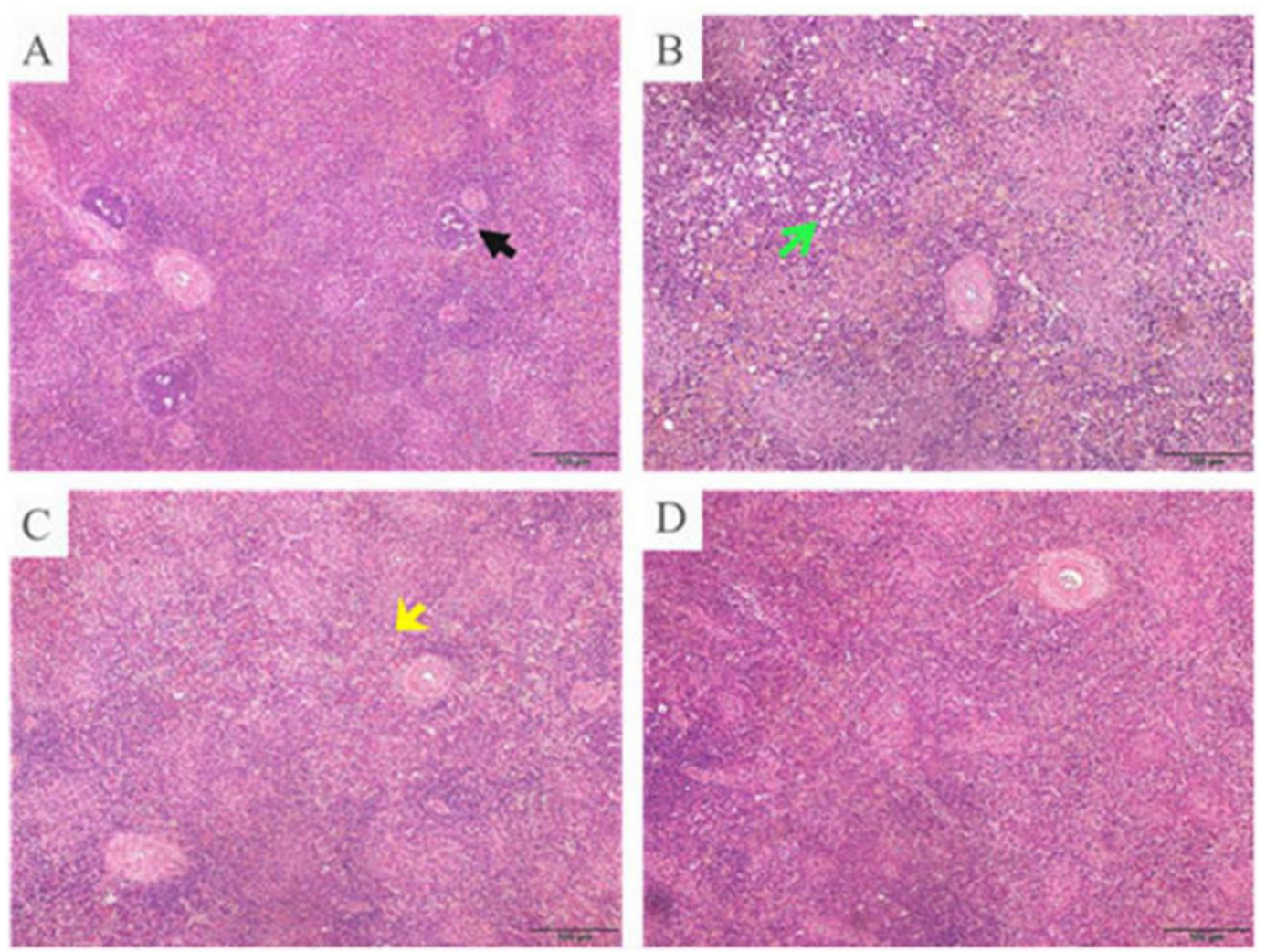

Figure 2. Pathological section of spleen (200×). (A) BC group; (B) IBDV group; (C) B87+IBDV group; (D) MFEC+B87+IBDV group. Black arrow is white pulp. Green arrow is tissue cavitation. Yellow arrow is diffuse bleeding. 

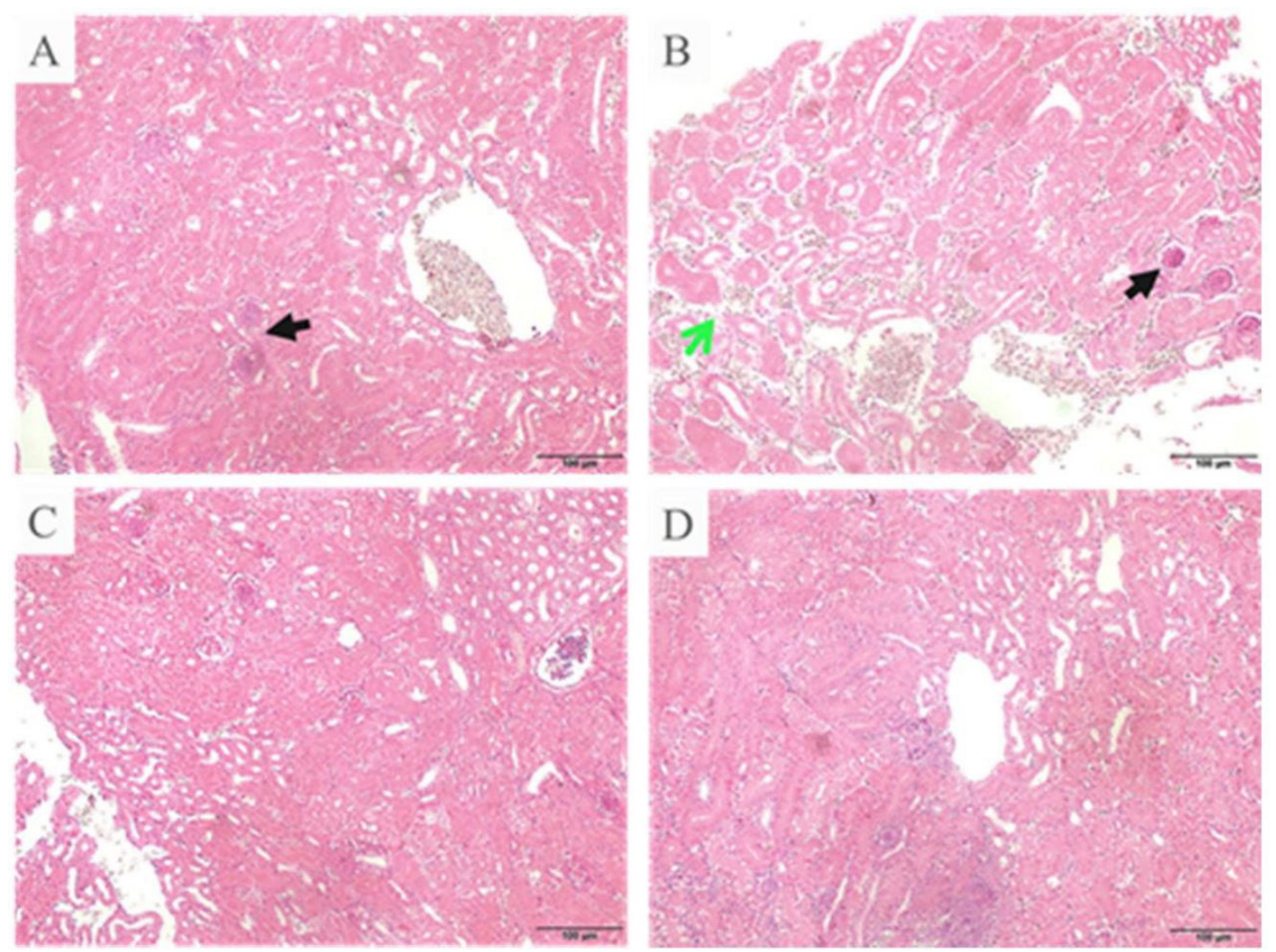

Figure 3. Pathological section of kidney (200×). (A) BC group; (B) IBDV group; (C) B87+IBDV group; (D) MFEC+B87+IBDV group. Black arrow is renal capsule. Green arrow is that the space between the tubules is enlarged and there are numerous blood cells.
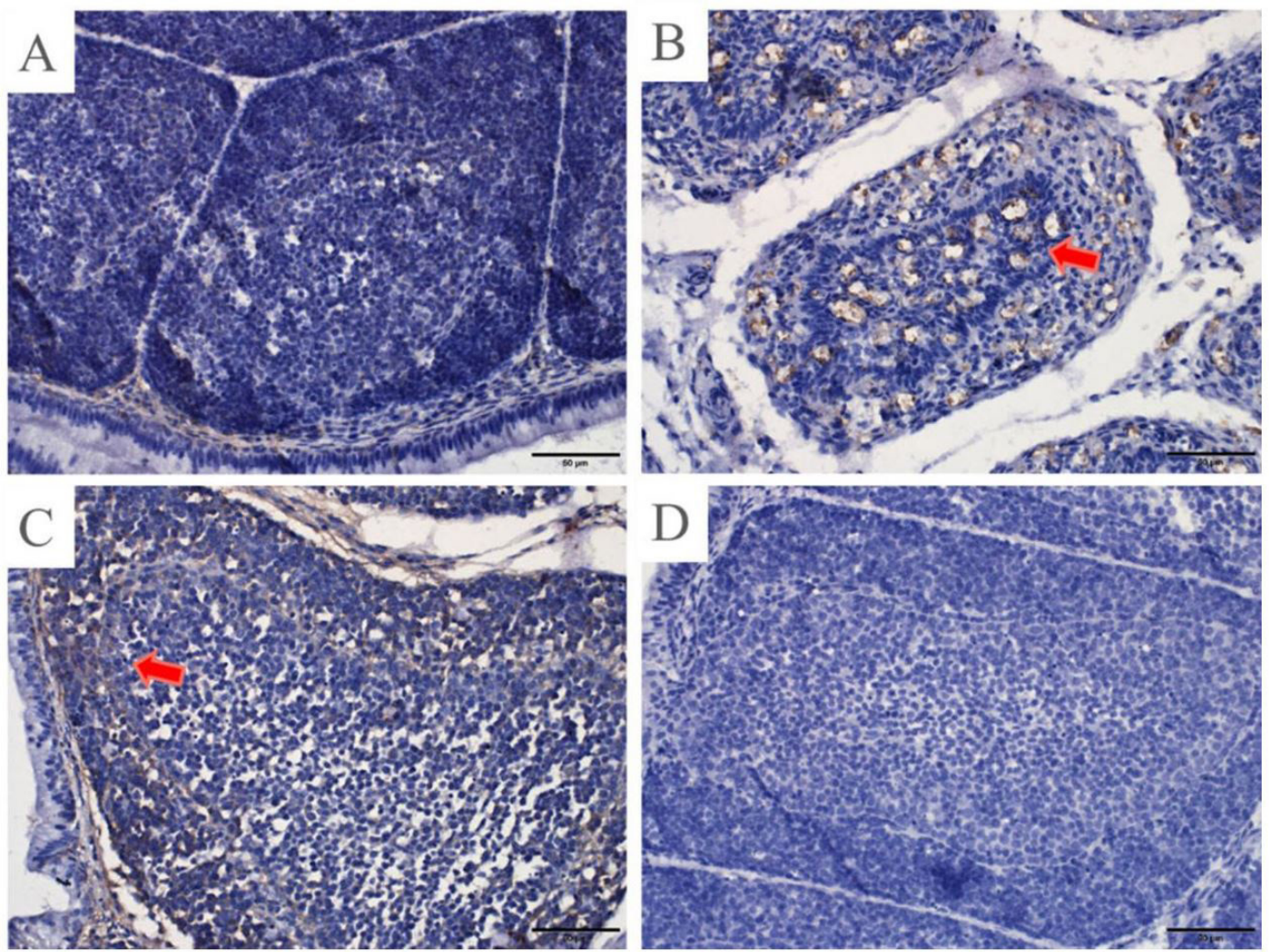

Figure 4. Expression of Bax in the bursa of Fabricius (400×). (A) BC group; (B) IBDV group; (C) B87+IBDV group; (D) MFEC+B87+IBDV group. Red arrow is yellow positive expression of Bax. 

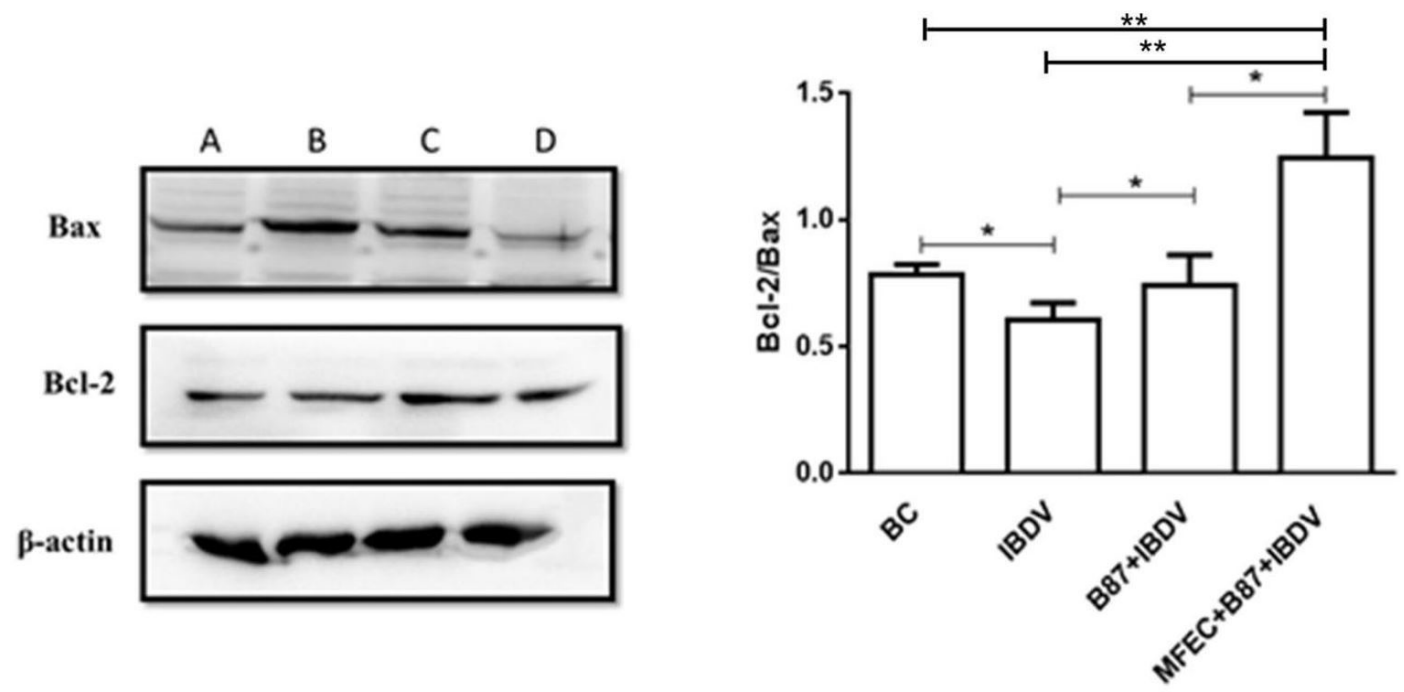

Figure 5. Changes of apoptotic proteins in each group. (A) BC group; (B) IBDV group; (C) B87+IBDV group; (D) MFEC+B87+IBDV group. ${ }^{\star}, P<0.05$. ${ }^{* *}, P<0.01$.

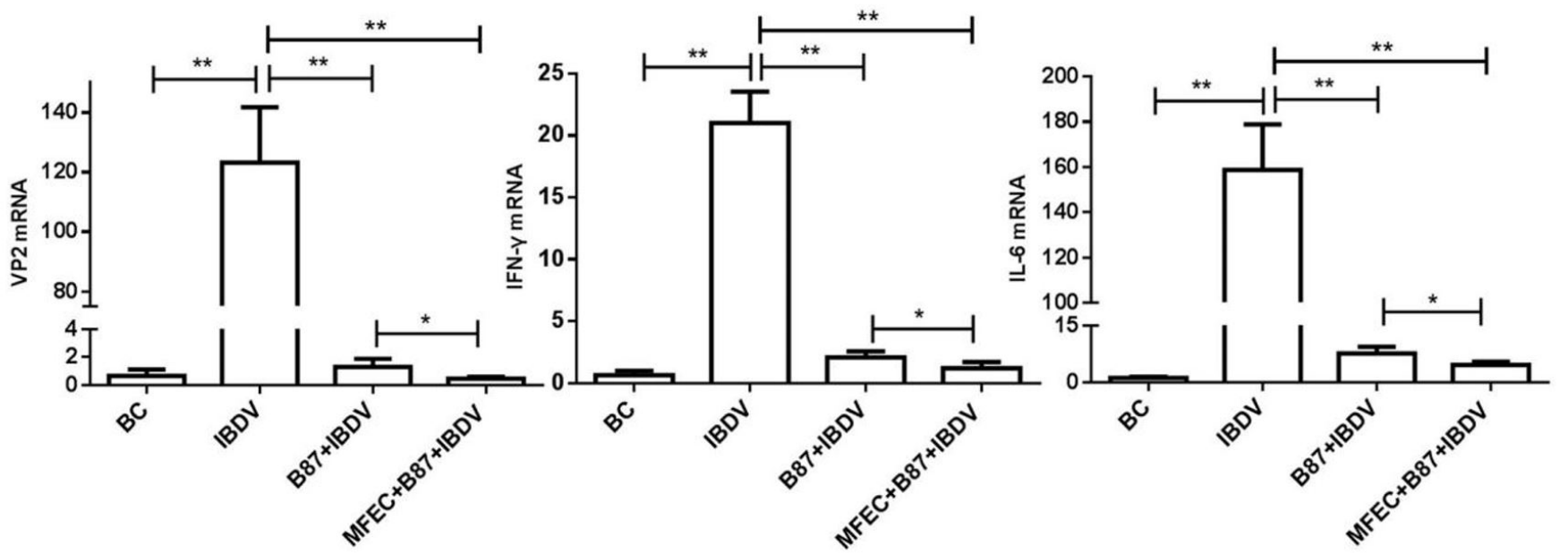

Figure 6. Expression of VP2, IFN- $\gamma$ and IL- 6 mRNA in bursa of Fabricius. ${ }^{\star}, P<0.05{ }^{* *}, P<0.01$.

\section{Discussion}

In this study, MFEC was demonstrated effectively to enhance the immune responses in chickens infected with bursal disease virus. Bursa of Fabricius, acknowledged as a humoral organ unique to chickens, is the main site of B cell development and maturation. Once infected with IBDV, bursa of Fabricius of chickens is always damaged, resulting in lower immunity and susceptibility to infection by other pathogens (Rautenschlein et al., 2002; Mahgoub et al., 2012). The thymus is the main site of T cell development and maturation and is related to cell-mediated immunity. The relative weights of the bursa of Fabricius and thymus are usually regarded as indices to elevate the immunity activity. In current study, clinical symptoms and the death appeared on the third day after infection in the IBDV group and the mortality rate was $40 \%$. Though there was no death in the B87+IBDV group, clinical symptoms such as loss of appetite, discharge of white watery stools, and atrophy of the bursa of Fabricius were observed. The chickens in MFEC+B87+IBDV group showed good defecation, diet, and mental status. Moreover, the antibody of MFEC+B87+IBDV group was significantly higher than that of other groups. The results of the comparison of morbidity, mortality, antibody levels and weight gain indicated that vaccine with MFEC can increase the protection efficiency of the vaccine, improve the production of antibodies, prevent viral infection, and also contribute to the body recover.

The elevation of ALT and AST in serum suggests liver lesions. In the IBDV group, ALT was 1.6 folds higher than that in the BC group, and AST was 0.7 folds higher than that in the $\mathrm{BC}$ group, indicating liver inflammation and lesions in IBDV group. As biochemical indicators of the B87+IBDV group and 
the MFEC+B87+IBDV group were in the normal range, the drug toxicity was excluded. TP is the sum of ALB and GLB and can be used to evaluate liver function, immune status and nutritional status. ALB, in plasma, maintains plasma osmotic pressure and synthesized by liver. GLB is involved in the body's immunity, promoting production of antibodies (Ping, 2016). The decrease of serum ALB also indicated that IBDV could affect the liver function. The level of GLB in B87+IBDV group and MFEC+B87+IBDV group was higher, suggesting higher antibody levels.

The typical pathological changes of IBD are bursal edema, hemorrhage and atrophy, kidney swelling, bleeding points on chest muscles and leg muscle (Rani \& Kumar, 2015). The necropsy and pathological section showed that the lesions of bursa of Fabricius, kidney and spleen in IBDV group and B87+IBDV group were serious and mild, respectively. No lesions were found in the MFEC+B87+IBDV group and the BC group, which indicated that the protection is reduced with incomplete vaccines, but MFEC can effectively improve the protection efficiency of the vaccine.

Viral infection can lead to a wide range of apoptosis, which would aggravate bursal damage and destroy the body's immunity (Tanimura \& Sharma, 1998). In the IBDV group, a large amount of Bax expressed in bursa of Fabricius, which was concentrated in the damaged structure, indicating that there was a large amount of apoptosis in bursa of Fabricius. In the B87+IBDV group, the expression of Bax was significantly reduced. There was nearly no Bax expression in the MFEC+B87+IBDV group and the BC group. The expression trend of $\mathrm{Bcl}-2$ in the four groups were similar. MFEC could effectively down-regulate the over expression of pro-apoptotic protein Bax caused by IBDV infection, increase the expression of $\mathrm{Bcl}-2$ and improve the apoptosis.

It has been reported that IFN- $\gamma$ can be used as adjuvants to enhance antigen presentation, $\mathrm{T}$ cell immune response and B cell humoral immune response (Lowenthal et al., 1998). The cytokine protein IL- 6 is secreted by Th2 cells and can promote the proliferation of $B$ cells and the production of antibody, which plays an important role in humoral immunity (Belardelli, 1995; Fan et al., 2012). Invasion of IBDV not only causes the depletion of B lymphocytes, but also induces the infiltration and mitosis of T lymphocytes, activation of macrophage, and up-regulates expression of IFN- $\gamma$ and IL-6 (Sharma et al., 2000). Here, MFEC could effectively reduce expression of IFN- $\gamma$ and IL-6, suggesting that MFEC could inhibit inflammation and body damage caused by excessive proliferation of $\mathrm{T}$ cells, and reduce the influence of vaccine and virus infection on the body.

\section{Conclusion}

In conclusion, MFEC combined with B87 can effectively prevent IBDV infection and promote immune response, thus reducing the risk of vaccine immune failure and promoting the recovery.

\section{Conflict of interest}

The authors declare that they have no competing interests.

\section{Funding}

This study is funded by the Natural Science Foundation of Jiangsu Province (BK20161368), University Natural Science Research Project of Jiangsu Province (16KJB230005), Qing Lan Project of Jiangsu Province; Animal Medicine Science and Technology Innovation Team of Jiangsu Agri-animal Husbandry Vocational College (NSF2021TC02).

\section{References}

Belardelli F. (1995). Role of interferons and other cytokines in the regulation of the immune response. APMIS: Acta Pathologica, Microbiologica, et Immunologica Scandinavica, 103(3), 161-179.

Chen, X.L., Yang, H.F., Jia, J.P., Chen, X.Y., Jiang, C.M., Wang, D.Y., \& Hu, Y.L. (2015) Chinese herbal medicine polysaccharide enhancing egg-yolk antibody titer of PRRS. Chinese Journal of Veterinary Science, 35, 1495-1500.

Delibaier, Saifuding, A., Yibulayin, M., \& Adelijiang, W. (2017). Effects of polysaccharides from ferula sinkiangensis root on proliferation of chicken peripheral lymphocyte and spleen lymphocyte in vitro. China Animal Husbandry \& Veterinary Medicine, 42(2), 601-606.

Dey, S., Chellappa, M. M., Pathak, D. C., Gaikwad, S., Yadav, K., Ramakrishnan, S., \& Vakharia, V. N. (2017). Newcastle disease virus vectored bivalent vaccine against virulent infectious bursal disease and newcastle disease of chickens. Vaccines, 5(4), 31. http://dx.doi. org/10.3390/vaccines5040031. PMid:28954433.

Fan, Y., Wang, D., Hu, Y., Liu, J., Han, G., Zhao, X., Yuan, J., Liu, C., Liu, X., \& Ni, X. (2012). Liposome and epimedium polysaccharidepropolis flavone can synergistically enhance immune effect of vaccine. International Journal of Biological Macromolecules, 50(1), 125-130. http://dx.doi.org/10.1016/j.ijbiomac.2011.10.008. PMid:22020157.

Ivanyi, J., \& Morris, R. (1976). Immunodeficiency in the chicken. IV. An immunological study of infectious bursal disease. Clinical and Experimental Immunology, 23(1), 154-165. PMid:177236.

Jackwood, D. J. (2017). Advances in vaccine research against economically important viral diseases of food animals: Infectious bursal disease virus. Veterinary Microbiology, 206, 121-125. http://dx.doi.org/10.1016/j. vetmic.2016.11.022. PMid:27916318.

Jia, J. P., Jin, L. Q., Chen, X. L., Zhou, Y., Zhang, D., \& Zhang, T. T. (2018). Research advances of effective components of sangdu oral liquid prepared with mulberry leaves and eucommia ulmoides, a novel compound prescription of traditional chinese veterinary medicinie. Animal Husbandry and Feed Science, 39, 9, 34-37.

Leng, C., Han, L. X., Yu, H. B., Luan, Y., Li, S. L., \& Qu, L. D. (2007). Determination of values of physiological and biochemical parameters in BWEL-SPF chickens at age of 10,40 and 60 weeks. Chinese Journal of Comparative Medicine, 2007, 12, 697-701.

Lowenthal, J. W., O’Neil, T. E., Broadway, M., Strom, A. D., Digby, M. R., Andrew, M., \& York, J. J. (1998). Coadministration of IFN-gamma enhances antibody responses in chickens. Journal of Interferon \& Cytokine Research: the Official Journal of the International Society for Interferon and Cytokine Research, 18(8), 617-622.

Mahgoub, H. A., Bailey, M., \& Kaiser, P. (2012). An overview of infectious bursal disease. Archives of Virology, 157(11), 2047-2057. http://dx.doi. org/10.1007/s00705-012-1377-9. PMid:22707044.

Matsushita, T. (2019). Regulatory and effector B cells: friends or foes? Journal of Dermatological Science, 93(1), 2-7. http://dx.doi. org/10.1016/j.jdermsci.2018.11.008. PMid:30514664.

Ping, Y. (2016). Collection and analysis of anatomical, physiological and biochemical data of $c z$-spf chicken. China: Harbin Normal University. 
Qin, Y., \& Zheng, S. J. (2017). Infectious bursal disease virus-host interactions: multifunctional viral proteins that perform multiple and differing jobs. International Journal of Molecular Sciences, 18(1), 161. http://dx.doi.org/10.3390/ijms18010161. PMid:28098808.

Rani, S., \& Kumar, S. (2015). Evaluation of infectious bursal disease virus stability at different conditions of temperature and $\mathrm{pH}$. Biologicals: Journal of the International Association of Biological Standardization, 43(6), 515-518.

Rautenschlein, S., Yeh, H. Y., Njenga, M. K., \& Sharma, J. M. (2002). Role of intrabursal T cells in infectious bursal disease virus (IBDV) infection: T cells promote viral clearance but delay follicular recovery. Archives of Virology, 147(2), 285-304. http://dx.doi.org/10.1007/ s705-002-8320-2. PMid:11890524.

Sharma, J. M., Kim, I. J., Rautenschlein, S., \& Yeh, H. Y. (2000). Infectious bursal disease virus of chickens: pathogenesis and immunosuppression. Developmental and Comparative Immunology, 24(2-3), 223-235. http://dx.doi.org/10.1016/S0145-305X(99)00074-9. PMid:10717289.

Shaw, I., \& Davison, T. F. (2000). Protection from IBDV-induced bursal damage by a recombinant fowlpox vaccine, fpIBD 1 , is dependent on the titre of challenge virus and chicken genotype. Vaccine, 18(28),
3230-3241. http://dx.doi.org/10.1016/S0264-410X(00)00133-X. PMid:10869768.

Spackman, E., Stephens, C. B., \& Pantin-Jackwood, M. J. (2018). The effect of infectious bursal disease virus-induced immunosuppression on vaccination against highly pathogenic Avian Influenza Virus. Avian Diseases, 62(1), 36-44. http://dx.doi.org/10.1637/11769110717-Reg.1. PMid:29620467.

Tanimura, N., \& Sharma, J. M. (1998). In-situ apoptosis in chickens infected with infectious bursal disease virus. Journal of Comparative Pathology, 118(1), 15-27. http://dx.doi.org/10.1016/S00219975(98)80024-8. PMid:9500235.

Wang, H., Shan, S., Wang, S., Zhang, H., Ma, L., Hu, L., Huang, H., Wei, K., \& Zhu, R. (2017). Fused IgY Fc and polysaccharide adjuvant enhanced the immune effect of the recombinant vp2 and vp5 subunits-a prospect for improvement of infectious bursal disease virus subunit vaccine. Frontiers in Microbiology, 8, 2258. http:// dx.doi.org/10.3389/fmicb.2017.02258. PMid:29184548.

Zhao, L. L., Wen, H. Q., Han, L. X., Zhao, L., Peng, Y. L., \& Li, S. L. (2018). Effects of different weeks of age and sex on the main organ coefficients, intestinal length and body size of SJ5-SPF chickens. Chinese Journal of Comparative Medicine, 28(6), 59-64. 